\title{
Un modèle d'association graminée-légumineuse : le mélange vesce (Vicia sativa L.)-avoine (Avena sativa L.)
}

\author{
Mustapha OUKNIDER \& Pierre JACQUARD (*) \\ Chaire d'Agronomie, E.N.A., B.P.S. 40, Meknès, Maroc. \\ (*) Unité de Biologie des populations et des peuplements, Centre Louis Emberger, C.N.R.S., B.P. 505I, \\ F 34033 Montpellier Cedex
}

\begin{abstract}
Les associations obtenues à partir de la variation des proportions relatives de 2 espèces créent des conditions variables, caractérisées par une modification des relations entre les individus du peuplement associé, et par là, de leurs comportements. La dynamique de croissance de la biomasse aérienne de 2 espèces (Vicia sativa et Avena sativa) a été étudiée au cours du cycle cultural. Une amélioration des états de croissance et de développement de la graminée associée a été mise en évidence. La vesce s'est montrée fortement concurrencée par l'avoine. Au niveau des associations, les interférences biologiques qui en résultent font apparaître des situations de compétition (avec surcompensation) au profit de l'avoine. Quand la proportion de la vesce est plus élevée que celle de l'avoine, la vesce supporte la concurrence et peut se montrer compétitive. La proportion relative seule a une grande signification dans la détermination du rendement de chaque espèce. L'intensité des relations varie suivant la proportion relative des espèces constituantes et selon l'apport d'eau.
\end{abstract}

Mots clés additionnels : Association vesce-avoine, série de remplacement, croissance, compétition.
A model of grass-legume association : vetch (Vicia sativa L.)-oat (Avena sativa L.).

The mixtures obtained by varying the relative proportions of 2 species are characterized by different relationships between individuals in the mixed stand, and, consequently, by different individual responses. The growth dynamics of the above-ground biomass of 2 species (Vicia sativa and Avena sativa) was studied throughout the cropping cycle. Growth and development of oats was improved in the association, while vetch was strongly depressed. The biological interactions resulted in a competitive situation (with over-compensation), to the advantage of oats. However, when the proportion of vetch was higher than that of oats, vetch could be competitive. Relative proportion was the only factor of great significance in determining the yield of each species. The intensity of the relationships changed with the relative proportions of the constituent species and with the water supply.

Additional key words : Vetch-oat association, replacement series, growth, competition.

\section{INTRODUCTION}

Les expériences sur les interférences dans les associations de plantes cultivées, et même d'animaux domestiques, peuvent avoir pour objet de répondre à 4 questions :

1. Comment les espèces en mélange se comportentelles vis-à-vis les unes des autres ? (TRENBATH, 1976).

2. Les espèces bénéficient-elles du mélange?

3. Les espèces sont-elles en compétition pour un pool commun de ressources? (TRENBATH, 1986).

4. Quels mélanges donnent le rendement optimum (TRENBATH, 1974).
Les 3 premières questions concernent principalement la concurrence (SPITTERS, 1983), alors que la $4^{\mathrm{e}}$ a surtout un intérêt agronomique. Ces différentes questions concernant les systèmes mixtes nécessitent une gamme de méthodes expérimentales et d'indices. Quelques-unes de ces méthodes ont été passées en revue par CONNOLLY (1986).

Les relations entre espèces, dans les mélanges binaires, en communautés artificielles, présentent 3 aspects :

1. Des aspects théoriques (TRENBATH, 1978) :

a) variations de densité à proportions fixes (les 
effets de la densité agissent alors sur la communauté et sur les individus),

b) variations de proportions à densités fixes (suivant le modèle de DE WIT, on teste dans ce cas si les espèces entrent en concurrence pour les mêmes ressources) (DE WIT, 1960 ; BAEUMER \& DE WIT, 1968),

c) motif de répartition spatiale (il a peu d'effet sur les performances globales, mais entraîne des différences dans les proportions des espèces),

d) processus en jeu (en utilisant la théorie de la croissance associée selon DE WIT, on peut identifier les facteurs limitants) ;

2. Des aspects agronomiques, mis en évidence par les nombreux exemples de croissance en association des espèces, que l'on peut rattacher à plusieurs types : mélanges quasi homogènes de céréales (méteil) ou hétérogènes (graminée-légumineuse), oasis (mélange à 3 strates) (BALDY, 1963 ; FRANCIS, 1986) ;

3 . Des aspects écologiques liés aux relations possibles entre diversité, stabilité et production.

Concernant les aspects agronomiques, les études sur les interférences des plantes en cultures associées sont liées directement ou indirectement à des processus d'alimentation particuliers à chaque espèce entrant dans la composition du mélange. En association, ces interférences se traduisent sur une espèce donnée, sous forme de réponses plastiques (JACQUARD, 1968, 1975), et le rendement moyen de chaque espèce est sous l'influence de la fréquence relative des 2 espèces et de la densité totale.

Les plantes dominées à une certaine période de leur vie, peuvent perdre leur faculté d'homéostasie et disparaître à la moindre limitation des disponibilités du milieu : la phase ultime de la réponse plastique est la mort de l'individu (DEMARLY et al., 1964). Cette mortalité est la cause de l'évolution du peuplement en place. Sous cet angle, la compétition peut être vue comme un moyen par lequel l'environnement ajuste l'équilibre entre les composants d'une association.

Dans les mélanges de légumineuses et d'espèces non légumineuses une relation particulière joue entre les composants puisque l'un d'eux bénéficie de 2 voies possibles de nutrition azotée (JACQUARD, 1977). Bien que l'association ne soit pas toujours bénéfique, il en est souvent ainsi (MAITRE, 1981; MAITRE et al., 1985). L'association graminée-trèfle blanc apporte de nombreux avantages. Les facteurs de variation de la production y sont connus (NOSBERGER, 1983). Le stade de croissance à la défoliation a une influence sur le trèfle. D'autres variables agissent : l'alimentation potassique qui favorise le trèfle, l'espèce de graminée, la variété de trèfle blanc. Les associations graminéeluzerne sont l'objet de nombreuses recherches et expérimentations (TALAMUCCI, 1976; ZANNONE et al., 1983 ; RoTILI et al., 1983 ; CRUZ, 1985). Beaucoup d'essais ont montré les avantages qu'elles apportent : la production annuelle est généralement plus importante avec les associations. Les conditions de sol sont plus strictes pour la luzerne pure. La répartition dans l'année de la production totale est meilleure. La recherche d'aptitudes variétales à l'association est à l'ordre du jour. Toutefois, la conduite d'une association est toujours très difficile car il faut maintenir un équilibre entre 2 constituants très différents. En effet, les caractéristiques intéressantes de l'association ne peuvent se manifester que si la légumineuse est présente en quantité suffisante. Un équilibre graminéelégumineuse doit être recherché selon l'objectif. Or, dans une association, les contributions absolues des espèces constitutives ne changent pas simultanément ou changent dans la même direction, engendrant ainsi une fluctuation dans l'équilibre de l'association. Certains aspects relatifs à l'écologie des associations graminée-légumineuse peuvent être clarifiés par l'étude des prairies naturelles. Enfin l'analyse des relations entre graminées et légumineuses a fait l'objet de modèles simplifiés, tels que celui de Ross et al. (1972).

Le présent travail s'inscrit dans le cadre d'un programme de recherche dont le but est de comprendre le fonctionnement de l'association vesce-avoine et se propose, à partir de l'élaboration de matière sèche par la plante, d'en déduire : la nature des interférences biologiques et d'étudier l'influence de la proportion relative des espèces en mélange sur le processus de compétition. Une partie des données concernant la production et la valeur nutritive ont déjà été présentées dans un article précédent (OUKNIDER \& JAC. QUARD, 1986).

\section{MATÉRIEL ET MÉTHODES}

\section{A. Conditions pédoclimatiques}

1. Sol: Les essais ont été conduits en plein champ durant 3 campagnes 1982, 1983 et 1984 ; ils étaient installés sur la parcelle d'essais de la Chaire d'Agronomie de l'Ecole Nationale d'Agriculture de Meknès au Maroc, sur un sol argilo-calcaire, peu profond, normalement pourvu en matière organique (2,2 p. 100), riche en $\mathrm{K}_{2} \mathrm{O}$ échangeable $(0,46 \mathrm{p} .1000)$ et fortement carencé en $\mathrm{P}_{2} \mathrm{O}_{5}(0,125$ p. 1000$)$, et dont le pH est de 8,0 .

2. Climat: La région de Meknès se caractérise par un climat semi-aride (classification d'Emberger). La pluviosité moyenne annuelle est de l'ordre de $560 \mathrm{~mm}$. Au cours des 3 années d'essais, les conditions climatiques ont différé essentiellement par la pluviosité (fig. 1).

\section{B. Conditions culturales}

\section{Matériel végétal}

On a utilisé :

- pour la graminée: l'avoine (Avena sativa L.) variété 153 , tutrice, demi-précoce, à tallage moyen, paille assez haute $(1,25$ à $1,35 \mathrm{~m})$, tige grosse et rigide, assez résistante aux rouilles et au charbon, résistante à la sécheresse hivernale et printanière. La rigidité des tiges est considérée comme un avantage pour les associations (vesce-avoine) où la céréale sert de tuteur à la légumineuse ;

- pour la légumineuse : la vesce (Vicia sativa L.), il s'agit d'une vesce de printemps, variété 6242, assez tardive et relativement résistante aux rouilles. 

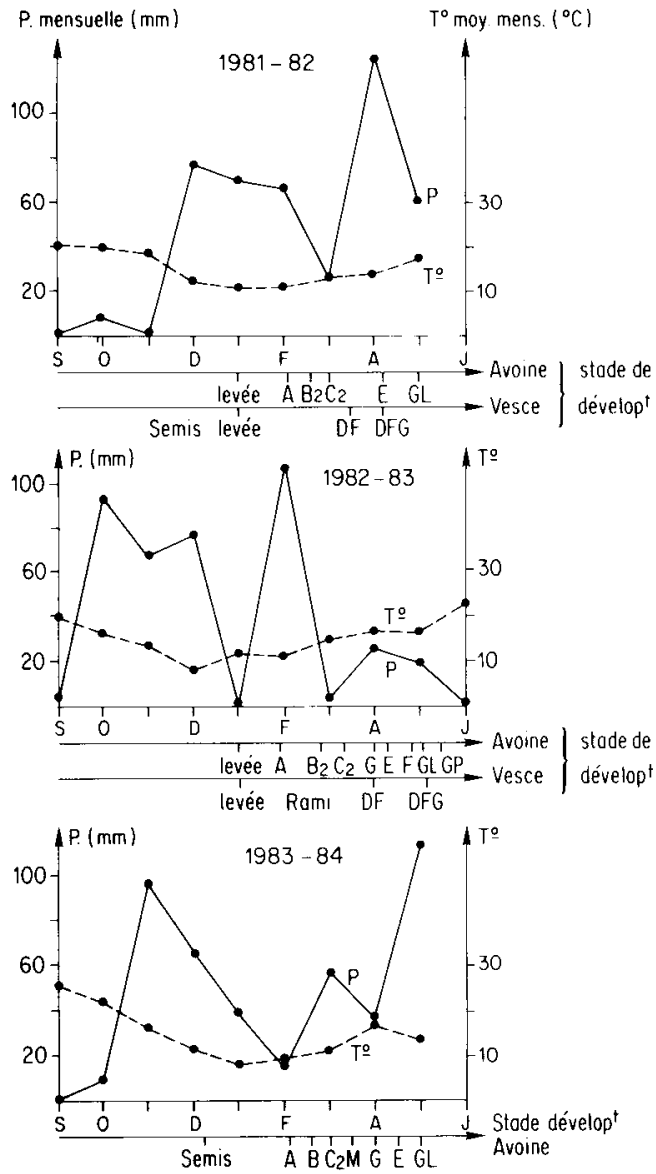

Figure 1

Conditions climatiques (pluviosité et température moyenne mensuelles) des 3 années d'expérimentations.

Climatic conditions (monthly rainfall and average temperature) for the 3 experimental years.

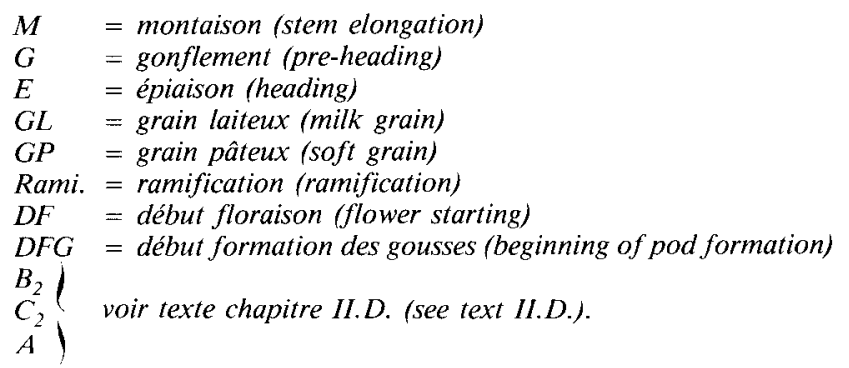

\section{Fertilisation}

Une fumure de fond à base d'un engrais binaire 026-26 a été épandue, après un travail du sol, à raison de 100 unités de $\mathrm{P}_{2} \mathrm{O}_{5}$ et 100 unités de $\mathrm{K}_{2} \mathrm{O}$. Pour l'azote 40 unités ont été apportées avant semis sur l'ensemble des traitements (1 unité $=1 \mathrm{~kg} / \mathrm{ha}$ ).

\section{Semis}

Il a été effectué entre la $1^{\text {re }}$ et la $2^{\mathrm{e}}$ décade de décembre ; pratiquement la pleine levée a eu lieu à la même date pour les 3 campagnes d'essais (vers le $1^{\text {er }}$ janvier) ; celle-ci a été notée une fois que 50 p. 100 des plantules avaient émergé et a permis de calculer les proportions réelles de chaque espèce. L'écartement entre les lignes était de $0,20 \mathrm{~m}$; pour l'association, les graines de vesce et d'avoine ont été localisées sur la même ligne. Sur la ligne, l'écartement est variable suivant les compositions testées. La densité de semis pour les mélanges et les cultures pures a été de 300 graines $/ \mathrm{m}^{2}$. La figure 2 indique les compositions réelles obtenues à la levée.

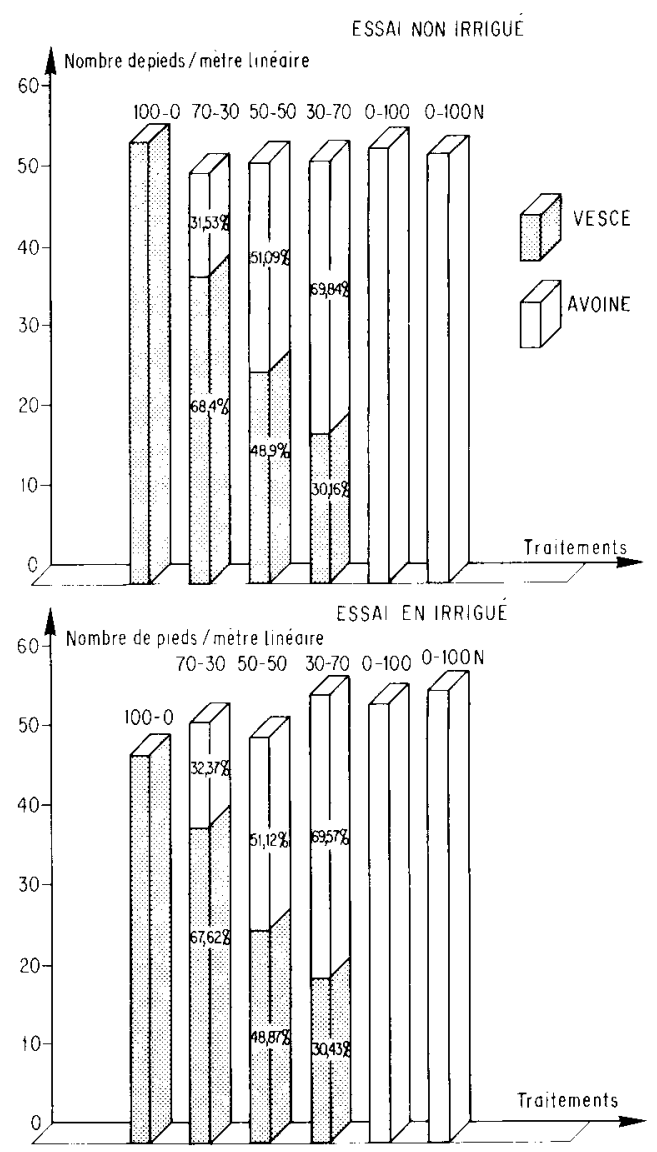

Figure 2

Composition moyenne des différents traitements en nombre de plantes à la levée (année 1983).

Average composition of the different treatments (plant numbers at seedling emergence) (year 1983).

\section{Dispositif expérimental}

Cinq traitements $100 \mathrm{~V}-0 \mathrm{~A}, 70 \mathrm{~V}-30 \mathrm{~A}, 50 \mathrm{~V}-50 \mathrm{~A}$, $30 \mathrm{~V}-70 \mathrm{~A}, 0 \mathrm{~V}-100 \mathrm{~A}$, visant à modifier simultanément les proportions relatives de chacune des espèces avec une densité totale constante ( 300 plantes $\left./ \mathrm{m}^{2}\right)$ ont été testés dans un dispositif en blocs randomisés à 5 répétitions. La taille de la parcelle élémentaire était de $8 \mathrm{~m}^{2}$. En 1983 et 1984 , les essais ont été conduits sous 2 régimes hydriques (sec et irrigué). Outre l'avoine en parcelle pure, un traitement avoine pure avec apport d'azote a été intégré au protocole des deux dernières campagnes, avec 60 unités d'azote apportées en 2 fois (au stade $\mathrm{A}$ et au stade $\mathrm{B}_{2}$ du brin maître de l'avoine).

\section{Observations et mesures}

Des prélèvements périodiques de placettes de deux lignes contiguës de 0,25 mètre linéaire chacune, sur l'ensemble des traitements de 3 blocs, ont permis de suivre la croissance des 2 espèces dans les différentes 
situations étudiées. Ils ont porté exclusivement sur la partie aérienne des plantes.

Huit prélèvements ont été effectués aux stades $\mathrm{A}$, $\mathrm{B}_{2}, \mathrm{C}_{2}$, gonflement, épiaison, floraison, grain laiteux et grain pâteux de la graminée. Pour le développement de l'avoine, l'échelle de JONARD et al. (1952) a été adoptée. Le stade $\mathrm{A}$ y est défini par la présence de stries blanches sur l'apex, le stade $B_{2}$ par l'apparition des glumelles sur les ébauches des épillets et $C_{2}$ est défini par l'apparition des ébauches d'anthères. Un suivi (sous la loupe binoculaire) au laboratoire des stades de l'avoine sur des plantes prélevées dans les parcelles correspondant aux différents traitements a permis de déterminer la date de chaque prélèvement, ceci pour les 3 premiers stades, les autres ont été précisés visuellement. On considère qu'un stade est réalisé lorsque 50 p. 100 des individus observés ont atteint le stade en question.

\section{E. Caractérisation des stades de la vesce par rapport à ceux de l'avoine}

Nous nous sommes servis des stades de l'avoine pour la détermination des dates de prélèvements, en raison des difficultés de détermination que présentent les stades de la vesce (chevauchement des phases de croissance).

\section{Avoine}

stade $A$

stade $\mathrm{B}_{2}$

stade $\mathrm{C}_{2}$

stade montaison

épiaison (E)

grain laiteux (GL)

grain pâteux (GP) gonflement $(\mathrm{G})$

\section{Vesce}

stade ramification (Rami)

émission de vrilles

boutons floraux (BF)

sortie $1^{\text {re }}$ fleur (DF)

floraison (F)

début formation des gousses (DFG)

formation des grains dans les gousses (FG)

Mis à part le stade épiaison pour lequel il a été noté un retard pour l'avoine associée par rapport à la culture pure, nous n'avons pas observé de décalage sensible pour une espèce donnée, quant à la réalisation d'un stade de développement, du fait des différents sance.

A la récolte, l'estimation des rendements a été faite sur $2 \mathrm{~m}^{2}$ par parcelle pour toutes les répétitions et a eu lieu au stade grain pâteux de l'avoine. A chaque date de prélèvement et pour chacune des espèces (association et culture pure), les mesures suivantes ont été effectuées : res ;

- matière sèche à l'étuve à $80{ }^{\circ} \mathrm{C}$ pendant 48 heu-

- surface foliaire ;

- évolution du peuplement sur des placettes fixes.

\section{RÉSULTATS}

Les données recueillies ont fait l'objet d'analyses de variance correspondant au dispositif expérimental traitements, malgré leur effet sur la cinétique de crois- adopté. Les effets bloc n'ont jamais été significatifs, ni l'interaction proportions $\times$ irrigation.

\section{A. Production}

\section{Effet de l'association sur la production de matière sèche de vesce et d'avoine}

Les courbes de la figure 3 traduisent l'évolution au cours du cycle de développement de la plante. Les biomasses correspondant aux modes de culture (fig. 3a) présentent des différences importantes par rapport aux témoins conduits en monoculture. $\mathrm{Au}$ début du cycle, les 2 espèces présentent le même comportement quel que soit le mode de culture et, à partir du stade début montaison de la graminée, l'association a un effet déprimant pour la vesce et un effet améliorant pour l'avoine. Au fur et à mesure que la vesce se développe, la contrainte créée par l'avoine augmente, jusqu'au stade épiaison où l'on atteint un seuil nuisible de très forte dépression de la vesce associée. Ceci se traduit (fig. 3a) par une diminution très marquée, voire même un arrêt de la croissance, ce qui suggère qu'à partir de ce stade la perte de biomasse (matière sèche/plante), à la suite de la mortalité foliaire, s'accentue chez les vesces en association, par rapport à la culture pure. En effet, à partir du stade
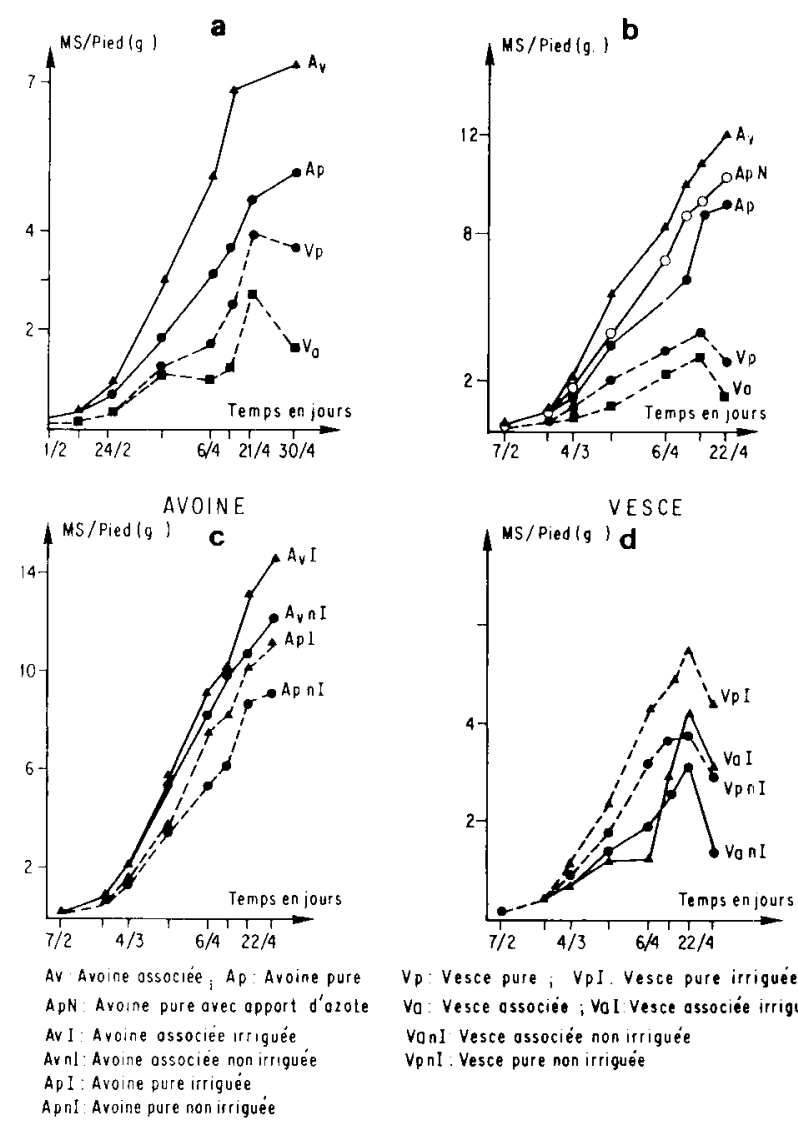

$V_{p}$ : Vesce pure; $V_{p} I$, Vesce pure irriguée $V_{0}$ : Vesce ossocieio; Vol Vesce associée irrigua VaaI Vesce associée non irriquée VpnI : Vesce pure non irriguée
Effets de l'association et de l'apport d'eau sur les états de croissance des plants de vesce et d'avoine en culture pure et en association (année 1983).

Effects of association and water supply on the growth stages of vetch and oat plants in pure stand and mixture (year 1983). 
montaison, la surface foliaire de l'avoine est maximale. La conséquence en est un ombrage important de la vesce ; dans les strates inférieures, la respiration de maintenance peut alors dépasser la photosynthèse.

Dans le même ordre d'idée, des durées phyllochroniques sensiblement plus longues chez la vesce en association par rapport à la culture pure ont été observées (OUKNIDER, 1985). La durée du phyllochrone 6 y a été de 72 et 83 degrés jours respectivement pour la vesce en pur et la vesce associée, ce retard s'amplifiant avec l'ordre de la feuille et la densité de peuplement. De son côté, MAITRE (1981) a noté sur trèfle violet associé au ray-grass d'Italie des durées de vie foliaire plus courtes.

Par opposition à la vesce, une inversion du phénomène s'observe pour l'avoine ; sa croissance est nettement améliorée par la présence de la vesce. A partir du stade $C_{2}$ du brin maître, la biomasse par plant d'avoine est significativement supérieure à celle de l'avoine en parcelle pure. Comparée à celle de l'avoine pure ayant reçu 60 unités d'azote (fig. 3b), une supériorité de l'avoine associée se dégage. Les conditions créées par l'association ont permis des états de croissance meilleurs que ceux entraînés par un apport d'azote sur culture pure. La densité biologique (ROTILI, 1979) est fortement diminuée pour l'avoine en conditions de compétition avec la vesce, puisque à même densité numérique globale qu'en culture pure la biomasse par plante est augmentée.

\section{Effet de l'apport d'eau sur la croissance de la vesce et de l'avoine en culture pure et en associa- tion}

L'apport d'eau a un effet favorable sur la croissance de l'avoine quel que soit le mode de culture (fig. 3c). L'irrigation a accentué l'effet déprimant sur la vesce en association entre le stade montaison et l'épiaison (fig. 3d) par rapport à la vesce associée irriguée. On assiste, sous irrigation, à une augmentation de la contrainte ombrage créée par l'avoine, suite à un tallage plus important et à une surface foliaire plus élevée qui se sont traduits au niveau des vesces par un nombre de feuilles fonctionnelles réduit et, par voie de conséquence, des bilans photosynthétiques de plus en plus faibles. En présence d'irrigation, la vesce a souffert d'un manque de lumière. Les plantes ombragées ont manifesté une morphologie typique: peu de rameaux et une hauteur plus élevée par rapport à la culture pure de vesce (tabl. 1) ; la lumière a été le facteur limitant pour la vesce en culture mixte.

TABLEAU 1

Effet de l'association et du régime hydrique sur certains paramètres de production mesurés sur des plants de vesce et d'avoine (essai 1984). Water supply and mixture effects on some production parameters measured on vetch and oat plants.

\begin{tabular}{|c|c|c|c|c|c|c|c|c|c|c|c|c|c|c|c|}
\hline & & \multicolumn{2}{|c|}{$7 / 2$} & \multicolumn{2}{|c|}{$15 / 2$} & \multicolumn{2}{|c|}{$27 / 2$} & \multicolumn{2}{|c|}{$11 / 3$} & \multicolumn{2}{|c|}{$26 / 3$} & \multicolumn{2}{|c|}{$4 / 4$} & \multicolumn{2}{|c|}{$13 / 4$} \\
\hline & & $\begin{array}{l}\text { non } \\
\text { irrigué }\end{array}$ & irrigué & $\begin{array}{l}\text { non } \\
\text { irrigué }\end{array}$ & irrigué & $\begin{array}{l}\text { non } \\
\text { irrigué }\end{array}$ & irrigué & $\begin{array}{l}\text { non } \\
\text { irrigué }\end{array}$ & irrigué & $\begin{array}{l}\text { non } \\
\text { irrigué }\end{array}$ & irrigué & $\begin{array}{l}\text { non } \\
\text { irrigué }\end{array}$ & irrigué & non & irrigué \\
\hline \multirow{3}{*}{ 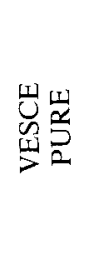 } & $\begin{array}{l}\text { Nbre total de feuille/pied } \\
(\text { NFT } / \text { P) }\end{array}$ & 21,7 & 22,0 & 28,1 & 27,8 & 34,8 & 39,5 & 60,5 & 67,4 & 68,78 & 78,25 & 78,6 & 82,0 & - & - \\
\hline & $\begin{array}{l}\text { Nbre rameau/plante } \\
(\mathrm{NR} / \mathrm{P})\end{array}$ & 5,2 & 5,5 & 6,2 & 6,52 & 7,5 & 7,0 & 8,5 & 8,9 & 9,84 & 8,0 & 11,4 & 14,0 & - & - \\
\hline & $\begin{array}{l}\text { Hauteur }(\mathrm{cm}) \\
(\mathrm{H})\end{array}$ & 12,7 & 13,0 & 17,0 & 17,0 & 27,0 & 35,0 & 40,0 & 52,0 & 58,0 & 78,0 & 81,0 & 88,5 & 89,0 & 110,2 \\
\hline \multirow{3}{*}{ 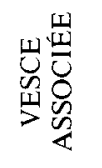 } & $\mathrm{NFT} / \mathrm{P}$ & 18 & 21,0 & 25,3 & 25,0 & 30,0 & 33,8 & 48,6 & 34,5 & 58,71 & 48,4 & - & - & - & - \\
\hline & $\mathrm{NR} / \mathrm{P}$ & 3,0 & 3,0 & 3,5 & 5,0 & 4,25 & 4,0 & 3,85 & 2,5 & 1,0 & 0,5 & 0,5 & - & - & - \\
\hline & $\mathrm{H}$ & 12,8 & 15 & 19,2 & 40,25 & 36,0 & 51,0 & 55,0 & 75,0 & 67,0 & 84,0 & 88,45 & 110,5 & 99,4 & 120,0 \\
\hline \multirow{3}{*}{ 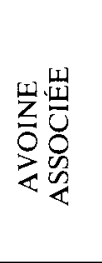 } & $\begin{array}{l}\text { Nbre feuille tige principale } \\
\text { (NF/BM) }\end{array}$ & 5,2 & 5,2 & 6,0 & 6,0 & 6,5 & 6,65 & 7,0 & 7,7 & 6,4 & 6,6 & - & - & - & - \\
\hline & $\begin{array}{l}\text { Nbre de talles/pied } \\
\text { (NT/P) }\end{array}$ & 3,5 & 3,5 & 3,5 & 3,5 & 5,4 & 6,6 & 7,6 & 8,9 & 6,1 & 6,0 & 6,1 & 5,1 & 4,3 & - \\
\hline & $\begin{array}{l}\text { Hauteur }(\mathrm{cm}) \\
(\mathrm{H})\end{array}$ & 23,2 & 23,2 & 33,0 & 34,0 & 46,0 & 47,0 & 61,6 & 67,0 & 74,0 & 110,3 & 100,0 & 123,6 & 120,0 & 139,0 \\
\hline \multirow{3}{*}{ 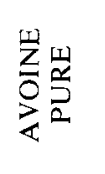 } & NF/BM & 5,3 & 5,3 & 5,5 & 5,5 & 6,0 & 6,5 & 7,2 & 7,8 & 8,5 & 8,5 & - & - & - & - \\
\hline & $\mathrm{NT} / \mathrm{P}$ & 2,0 & 2,5 & 2,5 & 3,0 & 4,0 & 3,1 & 3,3 & 4,7 & 3,3 & 3,3 & 3,5 & 2,9 & 3,2 & 3,5 \\
\hline & $\mathrm{H}$ & 27,5 & 27,5 & 35,0 & 45,0 & 50,0 & 65,0 & 72,5 & 86,5 & 97,0 & 120,0 & 123,0 & 140,0 & - & - \\
\hline \multirow{3}{*}{ 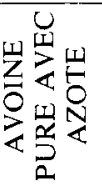 } & NF/BM & 5,3 & 5,3 & 6,0 & 6,0 & 6,9 & 6,3 & 7,5 & 7,6 & 6,5 & 6,6 & - & - & - & - \\
\hline & $\mathrm{NT} / \mathrm{P}$ & 3,5 & 3,5 & 3,9 & 4,0 & 3,3 & 3,2 & 3,2 & 3,7 & 3,6 & 3,4 & 4,2 & 3,4 & 3,8 & - \\
\hline & $\mathrm{H}$ & 27,5 & 27,5 & 33,8 & 33,2 & 46,3 & 46,1 & 68,2 & 78,1 & 84,9 & 112,8 & 96,4 & 135,8 & 107,2 & 147,2 \\
\hline
\end{tabular}




\section{Effet de la composition du mélange et de l'apport d'eau sur l'évolution de la surface foliaire}

La surface foliaire par plante est fortement corrélée à la biomasse par plante avant la montaison. Elle permet une analyse de l'indice foliaire $\left(\mathrm{m}^{2}\right.$ de feuille $/ \mathrm{m}^{2}$ de sol), lequel peut être un moyen de déterminer l'intensité de la compétition pour la lumière.

La surface foliaire d'un plant d'avoine en association est supérieure à celle de l'avoine pure. Elle est d'autant plus importante que le pourcentage de vesce dans le mélange est élevé. Au stade montaison, la surface foliaire est de $680,560,450$ et $245 \mathrm{~cm}^{2} /$ plante respectivement pour $70 \mathrm{~V}-30 \mathrm{~A}, 50 \mathrm{~V}-50 \mathrm{~A}, 30 \mathrm{~V}-70 \mathrm{~A}$ et $0 \mathrm{~V}-100 \mathrm{~A}$. La valeur maximale a été observée au stade montaison, stade à partir duquel les courbes de surface foliaire ont décroché suite au dessèchement des feuilles de base et à la régression des talles. L'effet des proportions du mélange vesce-avoine sur la surface foliaire de la vesce est symétrique de celui observé sur l'avoine : une augmentation de 20 p. 100 de la proportion de vesce entraîne une augmentation de $110-120 \mathrm{~cm}^{2}$ de la surface foliaire de l'avoine et une

TABLEAU 2

A) Evolution du pourcentage d'élimination des individus d'avoine ( $A$ et de vesce $(V)$ en culture pure et en association $(A+V=M)$ (essai 1983) (valeurs exprimées en \%).

Variation with time of the thinning percentage for individual oat $(A)$ and vetch $(V)$ in pure stand and in mixture $(A+V=M)$.

\begin{tabular}{lccccc}
\hline & & \multicolumn{2}{c}{$\begin{array}{c}\text { Phase levée } \\
\text { Gonflement }\end{array}$} & \multicolumn{2}{c}{$\begin{array}{c}\text { Phase gonflement } \\
\text { Grain pâteux }\end{array}$} \\
\cline { 2 - 6 } & & $\begin{array}{c}\text { Non irrigué } \\
\text { (NI) }\end{array}$ & $\begin{array}{c}\text { Irrigué } \\
\text { (I) }\end{array}$ & $\begin{array}{c}\text { Non irrigué } \\
\text { (NI) }\end{array}$ & $\begin{array}{c}\text { Irrigué } \\
\text { (I) }\end{array}$ \\
\hline $100 \mathrm{~V}-0 \mathrm{~A}$ & $\mathrm{~V}$ & 3,6 & 2,2 & 20,5 & 24,0 \\
\hline $70 \mathrm{~V}-30 \mathrm{~A}$ & $\mathrm{~V}$ & 4,9 & 2,7 & 30,0 & 38,0 \\
& $\mathrm{~A}$ & 5,2 & 1,3 & 3,5 & 4,0 \\
& $\mathrm{M}$ & 5,0 & 3,0 & 27,5 & 26,6 \\
\hline $50 \mathrm{~V}-50 \mathrm{~A}$ & $\mathrm{~V}$ & 4,0 & 6,0 & 35,8 & 40,0 \\
& $\mathrm{~A}$ & 4,4 & 0 & 5,0 & 3,2 \\
& $\mathrm{M}$ & 4,2 & 3,0 & 20,0 & 21,0 \\
\hline $30 \mathrm{~V}-70 \mathrm{~A}$ & $\mathrm{~V}$ & 6,0 & 4,5 & 38,6 & 41,0 \\
& $\mathrm{~A}$ & 4,0 & 3,4 & 5,2 & 3,4 \\
& $\mathrm{M}$ & 4,4 & 3,7 & 21,0 & 18,5 \\
\hline 0 V-100 A & A & 8,6 & 8,0 & 22,0 & 21,4 \\
\hline 0 V-100 A(N) & $\mathrm{A}$ & 3,0 & 6,7 & 18,4 & 14,1 \\
\hline \hline
\end{tabular}

B) Composition des mélanges au stade $A$ et à la récolte sous les 2 régimes hydriques (essai 1983) (en $\%$ du nombre de pieds total).

Mixture composition at stage $A$ and at harvest under the 2 water supplies (percent of total number of plants).

Composition au stade $\mathrm{A}$ Composition à la récolte en $\%$ en $\%$

\begin{tabular}{lcccc}
\cline { 2 - 5 } & $\begin{array}{c}\text { Non irrigué } \\
\text { (NI) }\end{array}$ & $\begin{array}{c}\text { Irrigué } \\
\text { (I) }\end{array}$ & $\begin{array}{c}\text { Non irrigué } \\
\text { (NI) }\end{array}$ & $\begin{array}{c}\text { Irrigué } \\
\text { (I) }\end{array}$ \\
\hline 70 V-30 A & $73-27$ & $75-25$ & $62-38$ & $65-35$ \\
\hline 50 V-50 A & $51-49$ & $52-48$ & $41-59$ & $43-57$ \\
\hline $30 \mathrm{~V}-70 \mathrm{~A}$ & $31-69$ & $29-71$ & $23-71$ & $24-76$ \\
\hline
\end{tabular}

même augmentation des proportions de l'avoine entraine une baisse de la surface foliaire de la vesce de $25 \mathrm{~cm}^{2}$. Contrairement à ce qui se passe pour l'avoine, l'association a un effet déprimant sur la surface foliaire des plants de vesce, elle est d'autant plus réduite que le pourcentage d'avoine dans le mélange est grand. Au stade montaison, la surface foliaire des vesces est de $240,180,155$ et $130 \mathrm{~cm}^{2} /$ plante respectivement, pour les traitements $100 \mathrm{~V}-0 \mathrm{~A}, 70 \mathrm{~V}-30 \mathrm{~A}$, $50 \mathrm{~V}-50$ A el $30 \mathrm{~V}-70 \mathrm{~A}$. Les indices de surface foliaire correspondant aux associations varient de 9,9 $(70 \mathrm{~V}-30 \mathrm{~A})$ à $10,6-10,7(50 \mathrm{~V}-50 \mathrm{~A}$ et $30 \mathrm{~V}-70 \mathrm{~A})$, contre 7,2 pour la culture pure de vesce et 7,4 pour celle d'avoine. La compétition pour la lumière est non seulement déterminée par la position des feuilles dans les différentes strates du couvert mais aussi par la contribution de chaque espèce à l'indice foliaire total, soit, ici, 38, 22 et 11 p. 100 pour la vesce, dans les associations où elle participe initialement, au semis, pour 70 , 50 et $30 \mathrm{p} .100$, ce qui donne une idée de l'intense compétition pour la lumière qu'elle subit.

La concurrence due à l'effet d'association engendre une simplification du végétal caractérisée par une modification dans l'apparition des feuilles (OUKNIDER, 1985), aspect qui sera développé par ailleurs, vu son intérêt.

L'effet de l'irrigation n'est pas le même selon que la vesce est en association ou en culture pure et il paraît évident qu'il s'agit d'une variation de densité biologique. En culture pure, la surface assimilatrice a augmenté sous l'effet de l'irrigation ; au stade montaison, elle est passée de 245 à $345 \mathrm{~cm}^{2} /$ plante ; alors qu'en association une tendance à la diminution se dessine à partir du stade montaison. L'irrigation de l'association a entraîné une chute de surface foliaire de la vesce, qui s'expliquerait, semble-t-il, par le fait que le traitement a favorisé chez l'avoine une forte production de feuilles et une intensification du tallage rendant la céréale beaucoup plus agressive vis-à-vis de la vesce.

\section{Effet de l'association et de l'apport d'eau sur les autres paramètres de production}

Parmi les composantes du rendement, le tallage de la graminée et la ramification de la vesce ont manifesté une grande plasticité. Pour l'essai non irrigué, le nombre de talles par plantes (tabl. 1), au stade montaison, est en moyenne de 3,3 et 7,6 resyectivement pour l'avoine pure et l'avoine associée. Au stade correspondant de l'avoine, le nombre de rameaux par plant à la montaison est de 8,5 contre 3,5 respectivement pour la vesce pure et la vesce en association, et, à partir du stade début gonflement, la ramification est pratiquement nulle. L'association a entraîné chez la vesce une simplification en la réduisant, à partir du stade gonflement à la seule tige principale, l'ensemble des rameaux ayant régressé (tabl. 1). La vesce en association, au lieu d'émettre des rameaux en abondance, comme en culture pure, tend dans la parcelle d'avoine à acquérir la hauteur de son compétiteur. Pour l'avoine, le tallage est nettement favorisé en association par rapport à la culture pure ce qui se traduit par une surface assimilatrice importante. C'est l'étude de ces mécanismes qu'il faudra développer davantage car ils permettront d'analyser les modifications de struc- 
ture du peuplement en vue d'une modélisation de l'interception de la lumière par chacun des composants.

\section{B. Evolution du peuplement}

L'évolution des divers peuplements testés a été suivie sur des placettes fixes par des contrôles périodiques depuis la pleine levée. Les pieds soumis au contrôle ont été bagués. Quatre placettes de 1 mètre linéaire par traitement ont été choisies de manière à représenter les compositions théoriques de départ. Il se dégage des résultats obtenus que les peuplements de départ, illustrés par les histogrammes de la figure 2, ont évolué au cours du cycle cultural. Ainsi, les résultats du tableau 2, exprimant l'évolution du pourcentage d'élimination en nombre de pieds de vesce et d'avoine en culture pure et en association, montrent que : durant la phase levée-gonflement de la graminée, la perte en nombre de pieds est pratiquement négligeable sous les 2 régimes hydriques, quelle que soit la composition considérée. L'essentiel des disparitions a été enregistré durant la phase gonflement-grain pâteux. Il ressort également de ces résultats que l'élimination des pieds de vesce est plus importante en association qu'en culture pure. Elle a varié de 30 à 41 p. 100 selon les compositions testées, alors qu'elle est faible chez l'avoine associée. Pour les mélanges, la perte est d'autant plus importante que le mélange est riche en avoine. Il semblerait que la verse observée pour le traitement le plus riche en vesce soit en grande partie à l'origine de la disparition des pieds. Cependant, la perte observée dans le traitement $30 \mathrm{~V}-70 \mathrm{~A}$, d'une part, et l'élimination des pieds de vesce plus importante en association qu'en culture pure, d'autre part, montrent que la verse est loin d'être la seule cause. La mortalité est due aussi à une compétition entre les 2 espèces, comme le confirment les situations de compétition mises en évidence à partir du stade montaison de l'avoine (cf. paragraphe A).

Cette élimination est la résultante de la contrainte de l'avoine agissant sur la morphologie des individus de vesce associés. Ceci montre que l'évolution du peuplement s'annonce longtemps à l'avance par une déformation plastique (réponse du phénotype) due à la contrainte créée par l'environnement biologique (partenaire) et semble être ici un phénomène continu contrairement à ce qui a été signalé par GUY (1965) lequel a rapporté que l'élimination est un phénomène discontinu et indépendant de l'âge.

Par ailleurs, les résultats du tableau 2B montrent que l'élimination des pieds sous la pression de compétition a entraîné une modification dans la composition du mélange entre le stade $\mathrm{A}$ et la récolte. Les proportions relatives des 2 espèces ont évolué dans le même sens mais avec des intensités différentes engendrant alors un déséquilibre dans les compositions des mélanges, au profit de l'avoine; la conséquence en est un faible pourcentage de vesce en poids sec au niveau du rendement global. Il convient de signaler que le faible pourcentage en poids sec de la vesce à la récolte (OUKNIDER \& JACQUARD, 1986) résulte de l'élimination des pieds de vesce, d'une part, et de l'effet du composant avoine sur les états de croissance et de développement des individus de vesce associés survivants (déformations plastiques) d'autre part.

\section{Interférences biologiques}

L'association de 2 espèces vise une amélioration quantitative et/ou qualitative de la production. Un effet bénéfique est alors recherché. Cependant, il n'est pas toujours obtenu et de nombreuses situations biologiques peuvent en être à l'origine.

Les différentes situations rencontrées peuvent être regroupées selon JACQUARD $(1975,1977)$ en 2 classes :

\section{- l'antagonisme :}

- opposition : les 2 individus sont désavantagés par l'association ;

- compétition : 1 individu profite aux dépens de l'autre ;

- la coopération: les 2 sont avantagés par l'association par rapport à leurs cultures pures respectives.

Plusieurs critères permettent de définir la nature de ces situations : le rendement global de l'association et des cultures pures, le rendement de chaque partenaire en association et en culture pure.

Le rendement de chaque partenaire et de l'association donne une idée de la production totale et de la part prise par chaque constituant dans l'élaboration du rendement. L'évaluation se fait par rapport à la culture pure. Le rendement relatif de chaque constituant de l'association se définit par rapport à sa culture pure :

$$
r_{i}=Y_{i / j} / Y_{i i}
$$

avec :

$\mathrm{r}_{\mathrm{i}}=$ rendement relatif,

$\mathrm{Y}_{\mathrm{i} / \mathrm{j}}=$ rendement en association,

$\mathrm{Y}_{\mathrm{ii}}=$ rendement en culture pure.

L'observation des réponses plastiques (fig. 4) à chaque stade de développement met en évidence l'existence de 2 types de relations dont l'intensité et la durée varient selon la composition du mélange au semis, laquelle induit une variation de la densité biologique déjà signalée par ZANNONE et al. (1986) :

- une période où l'effet de l'association est bénéfique pour l'un des composants. Cette situation de coopération unilatérale continue jusqu'au stade début montaison pour le traitement riche en vesce $(70 \mathrm{~V}$ $30 \mathrm{~A}$ ), alors qu'elle s'estompe dès le stade $\mathrm{B}_{2}$ du brin maître de l'avoine et même avant, respectivement, pour les compositions (50 V-50 A et $30 \mathrm{~V}-70 \mathrm{~A})$, laissant la place à une situation de compétition ; ces stades initiaux de "coopération » ont la même signification que les situations transitoires de coopération trouvées dans quelques cas par ZANNONE et al. (1986) : moments de passage d'une situation à l'autre $($ de +- à -+$)$ ou d'inertie initiale ;

- à partir du stade montaison, l'existence généralisée de situations de compétition devient la règle au niveau de tous les mélanges. Les situations fluctuent ; elles évoluent avec le stade de développement et les conditions environnementales et culturales.

La présence d'effets bénéfiques pour l'avoine et 

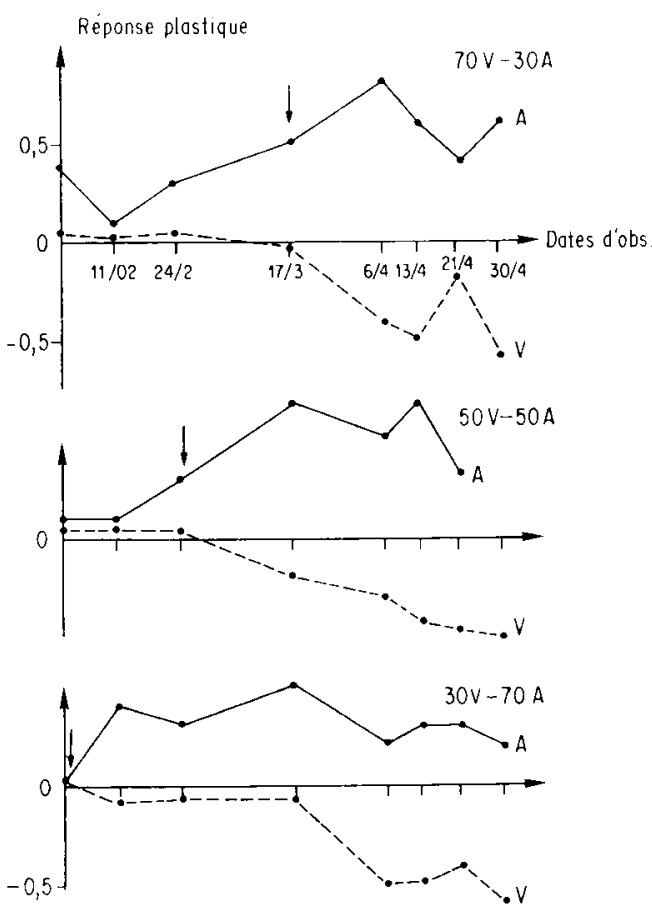

Figure 4

Effets des proportions sur la nature des relations de voisinage tessai 1983), réponses plastiques, en fonction du temps.

Proportion effects on the type of neighbour relationship (1983 experiment).

Réponse plastique $=\underline{\text { Performance en association }- \text { Performance en pur }}$ Performance en pur

Plastic response $=\frac{\text { Performancein association }- \text { Performance in purestand }}{\text { Performance }}$ Performance in pure stand

La flèche indique le point de départ de la compétition.

The starting of competition is indicated with an arrow.

d'effets négatifs sur la vesce, d'une part, et l'absence d'avantage quantitatif au niveau global par rapport à la culture pure la plus productrice, d'autre part, suggèrent que le comportement du mélange est du « type compensateur ". L'accroissement de biomasse d'une plante d'avoine par rapport à sa culture pure compense la décroissance accusée par la vesce associée par rapport à la culture pure.

Les courbes de la figure 5 ( $a$ et $b$ ) montrent que les situations évoluent avec l'âge des plantes, la composition du mélange et l'apport d'eau. A faible pourcentage d'avoine (70 V-30 A), la vesce supporte la concurrence et peut se montrer compétitrice (fig. 5a-1) et son pourcentage en poids sec dans le rendement global atteint des valeurs de 20 p. 100 (OUKNIDER \& JACQUARD, 1986). Toutefois au stade montaison, on observe une inversion du phénomène : un avantage pour l'avoine et un effet déprimant pour la vesce.

Dans l'essai non irrigué, sous l'effet de l'épuisement des réserves en eau du sol, la vesce n'arrive plus à supporter la concurrence de l'avoine. Il en découle que les 2 plantes n'ont pas la même capacité à extraire l'eau du sol. En irrigué, par contre, on observe pour la même composition à partir du même stade une situation de coopération (fig. 5b-1). Sous l'effet de l'irrigation, les rendements relatifs des 2 espèces sont a

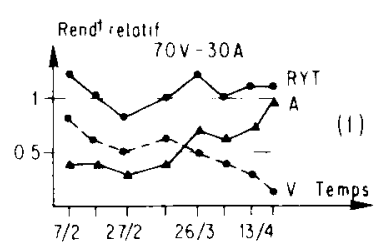

b
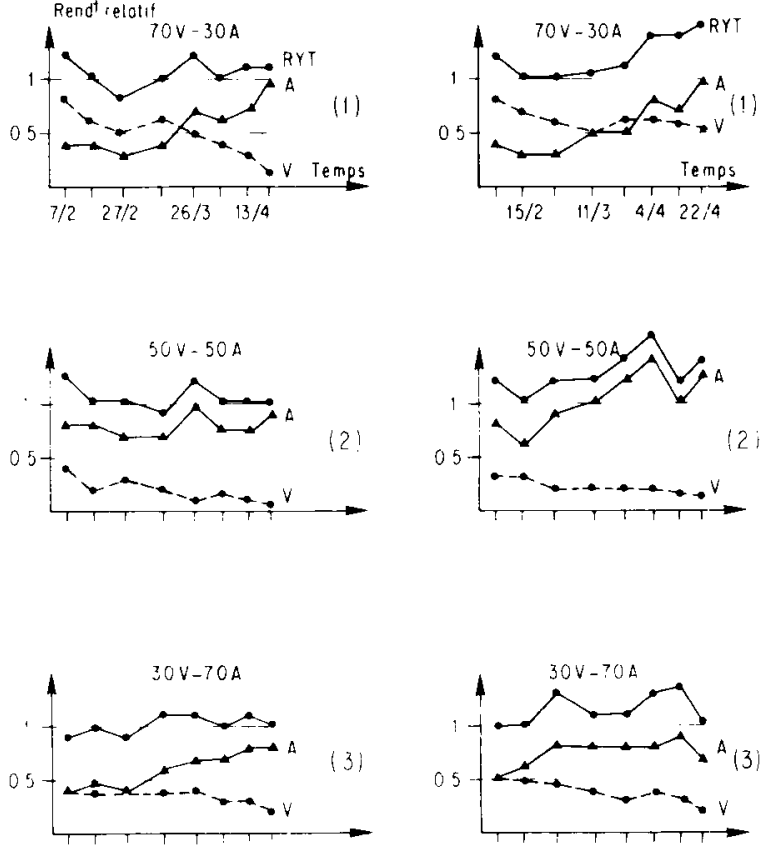

$\therefore$ Aroin

- nom irigue

-..- Vesce

b. Irrigue

-.-.-. Somme des rendements relotits IRYT!

Figure 5

Effets de l'apport d'eau et de la composition du mélange sur l'évolution des rendements relatifs et de leur somme (RYT) (essai 1984).

Water supply and mixture composition effects on the time variation of relative yields and relative yield totals $(R Y T)$ (1984 experiment).

supérieurs ou égaux à 0,5 indiquant que la compétition est plus équilibrée entre les 2 espèces. Par contre, la composition ( $30 \mathrm{~V}-70 \mathrm{~A}$ ) connaît au début du cycle une situation d'opposition (fig. 5a-3) suivie d'une situation de compétition avec surcompensation. L'irrigation a permis avec la même composition d'avoir une situation de compétition avec compensation stricte au début du cycle, suivie à partir du stade $\mathrm{B}_{2}$ du brin maître d'une situation de compétition avec surcompensation. Dans l'essai non irrigué, les 2 espèces ont souffert d'un manque d'eau dès le début du cycle, mais les résultats montrent que la vesce est plus fortement concurrencée si les réserves hydriques sont faibles.

Les résultats obtenus en 1983 (fig. 6), dans des conditions climatiques (fig. 1), en particulier la composante pluviosité, différentes, montrent un avantage des associations à certains stades de développement. On constate des situations de compétition avec surcompensation à partir du stade gonflement jusqu'au stade grain pâteux alors qu'au stade A, il n'y a pas d'interférence entre les 2 composants et qu'aux stades $\mathrm{B}_{2}$ et $\mathrm{C}_{2}$, il y a compétition avec compensation stricte. L'avantage des associations, observé dans certains essais est la conséquence des situations d'interférences. Selon ZANNONE et al. (1983) et ROTILI et al. (1983), seules les situations de coopération et de compétition avec surcompensation sont en mesure de conduire à des mélanges dont la production est supérieure à celle du meilleur composant. L'avantage des associations s'explique par les effets de surcompensation 
A _ ESSAI NON IRRIGUE (1983)
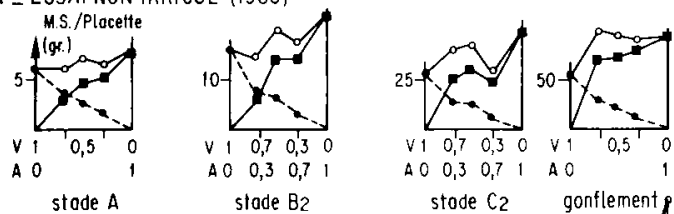

stode $\mathrm{C}_{2}$
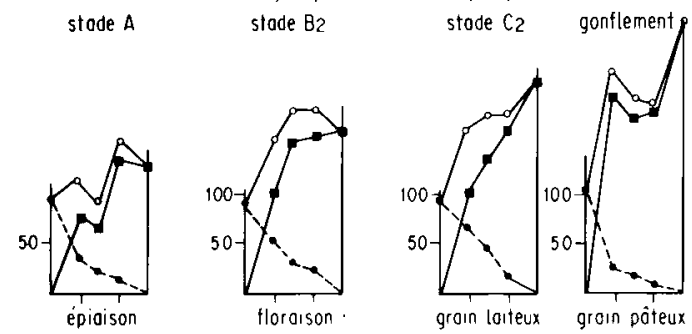

B - ESSAI IRRIGUÉ (1983)
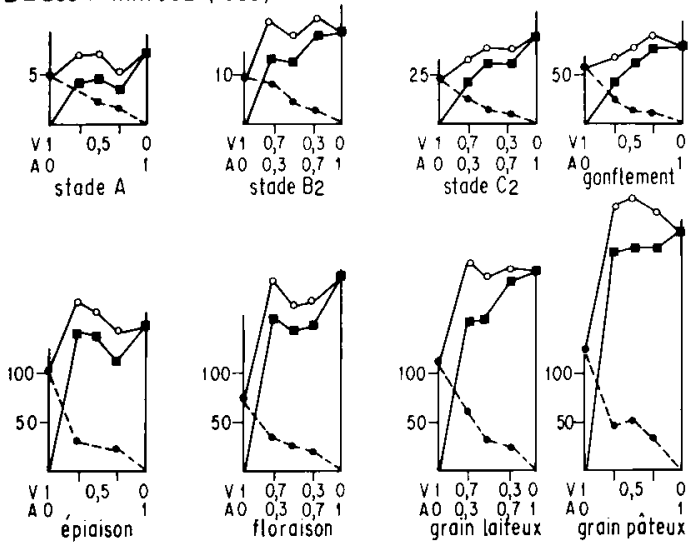

Figure 6

Résultats des différentes situations entraînées par les effets de voisinage (série de remplacement, essai 1983).

Avoine : Vesce :

Association : 0

Consequences of the different situations resulting from neighbour effects (replacement series, 1983 experiment).

(dans le cas de compétition) qui, en se cumulant les uns aux autres, conduisent l'association à produire plus que la moyenne des 2 cultures pures $\left(\mathrm{Y}_{\mathrm{ii}}+\mathrm{Y}_{\mathrm{jj}}\right) / 2$ ou mélange théorique et parfois plus que la meilleure des 2 cultures pures.

\section{DISCUSSION ET CONCLUSION}

Les essais réalisés sur vesce-avoine, en conditions de plein champ, au cours de 3 campagnes, ont montré que la vesce et l'avoine ont des comportements différents selon qu'elles sont en culture pure ou en association. Au début du cycle, la production par plante pour les 2 espèces en association est équivalente à celle de leur monoculture, et, à partir du stade montaison de la graminée, l'association a un effet déprimant sur la vesce et un effet améliorant pour l'avoine. Au fur et à mesure que la plante se développe, la contrainte ombrage créé par l'avoine atteint au stade épiaison un seuil de nuisance conditionnant fortement la dépression de la vesce.

Les états de croissance et de développement de l'avoine ont été notablement améliorés par la présence de la vesce : la matière sèche par plante est affectée de façon significative par la composition du mélange au semis. Cet effet bénéfique du partenaire sur les états de croissance de la graminée confirme bien d'autres résultats (FAUCONNIER, 1982 ; ARNAUX \& NiqueUX, 1982 ; HAYNES, 1980). Les courbes de croissance de l'avoine associée montrent une supériorité par rapport à l'avoine pure fertilisée (60 u. d'azote). Il se dégage, qu'à même densité numérique, les conditions créées par l'association ont permis des états de croissance meilleurs que ceux liés à un apport de 60 unités d'azote. Une telle observation amène à se demander s'il y a un enrichissement du milieu en azote par la légumineuse, par voie directe ou indirecte, ou s'il s'agit simplement de la particularité de la légumineuse qui présente 2 voies de nutrition quant à son alimentation en cet élément. Plusieurs travaux ont rapporté que lorsqu'une légumineuse est associée à une graminée, en principe beaucoup plus compétitive vis-à-vis de l'azote minéral, l'assimilation sera limitée et, de ce fait, elle fixera plus d'azote atmosphérique (HAYNES, 1980 ; CHALAMET et al., 1983). D'autres auteurs ZANNONE et al. (1983), ROTILI et al. (1983) ont conclu par contre que l'avantage des graminées en association pourrait être dû à des périodes de croissance différentes des 2 espèces. TRENBATH (1974) considère un autre mécanisme possible, tels que des espaces disjoints dans l'utilisation des ressources, liés aux profondeurs racinaires spécifiques.

L'irrigation a rendu l'avoine plus compétitive vis-àvis de la vesce par suite d'un nombre de talles par plante plus élevé et d'une plus grande surface foliaire ce qui se traduit par une diminution de l'énergie lumineuse arrivant au niveau des vesces. Les plants de vesces ombragés ont manifesté une morphologie typique : peu de rameaux et une hauteur plus grande par rapport à la vesce pure. L'évolution observée du peuplement est apparue après que la contrainte créée par l'avoine se soit traduite sur la morphologie des individus de vesce associée (simplification du végétal). Les proportions reiatives des 2 espèces ont évolué dans le même sens avec toutefois des intensités différentes engendrant alors un déséquilibre dans les compositions des mélanges testés au profit de l'avoine. Le faible pourcentage de vesce en poids observé à la récolte (OUKNIDER \& JACQUARD, 1986) résulte de l'élimination des pieds de vesce, d'une part, et, d'autre part, de l'effet du composant avoine sur les états de croissance et de développement des vesces associées (déformations plastiques).

Pour ZANNONE et al. (1985 et 1986) la nature des interférences interspécifiques se révèle identique à celle des interférences intraspécifiques et ces interférences sont interprétables sur la base de l'hypothèse de la «densité biologique » (densité numérique pondérée par la vigueur des plantes) (RoTILI, 1979). Dans les associations graminée-légumineuse, pérennes, les situations de compétition avec surcompensation proviennent alternativement de l'avantage d'une espèce ou de l'autre, en fonction de leur précocité ou de leur cycle biologique. Les situations de coopération sont rares et, quand elles surviennent, elles sont de courtes durées, transitoires. L'intensité des relations varie avec l'âge des plantes, les proportions relatives des composants et l'apport d'eau. La proportion relative, seule, a une grande signification dans la détermination 
du rendement relatif et de la composition du melange à la récolte. Quand la densité de la vesce est plus élevée que celle de l'avoine, la vesce supporte la concurrence et peut se montrer compétitive. Les résultats obtenus suggèrent que, dans les conditions de plein champ, les relations de compétition seront influencées durant l'établissement par la composition au semis. A partir du stade montaison, date à laquelle la contrainte lumière se fait sentir sur les individus de vesce, la proportion relative de chaque espèce sera contrôlée par la capacité de chacune d'elle à absorber l'eau disponible dans les horizons racinaires.
Une étude sur les mécanismes physiologiques est actuellement en cours à l'aide du modèle vesce-avoine.

Reçu le 10 décembre 1986. Accepté le 7 octobre 1987.

\section{REMERCIEMENTS}

Nous remercions G. Lemaire, J.-P. Maitre, P. Rotill.l et L. ZANNONE pour leur contribution critique à ce manuscrit.

\section{RÉFÉRENCES BIBLIOGRAPHIQUES}

\begin{abstract}
Arnaux R., Niqueux M., 1982. Possibilités d'association du trèfle violet avec différentes graminées en moyenne montagne. Fourrages, $89,3-27$.

Baeumer K., de Wit C. T., 1968. Competitive interference of plant species in monocultures and mixed stands. Neth. J. agric. Sci., 16, 103-122.

Baldy Ch., 1963. Cultures associées et productivité de l'eau, In : L'eau et la production végétale, I.N.R.A., Paris, 308-348, et Ann. agron., 14 (4), 499-534.
\end{abstract}

Chalamet A., Audergon J. M., Maitre J. P., 1983. Concurrence entre une graminée (Lolium multiflorum Lamk.) et une légumineuse (Trifolium pratense L.) : utilisation de $\mathrm{N}^{15}$ pour l'étude de la nutrition azotée. Oecol. Plant., 4 (18), 2, 12-33.

Connolly J., 1986. Importance and measurement of mixture effects in grazing systems. In : Grazing Research at Northern latitudes, $\mathrm{O}$. Gudmundson (ed.), NATO ASI Ser., A, Life sciences, Vol. 108, Plenum Press, N.Y., 323-333.

Cruz P., 1985. Relations de compétition dans une association luzerne-dactyle : étude des dynamiques de la croissance et des prélèvements d'azote. Thèse Doct. Ing., Ecologie Végétale, Univ. ParisSud, Orsay, 132 p.

Demarly Y., Guy P., Chesneaux M. T., 1964. Analyse préliminaire de la compétition chez les luzernes. Ann. Amélior. Plant., 14 (2), 131-155.

Fauconnier D., 1982. Avantage de l'association graminées-légumineuses et principes de fertilisation. Fourrages, 89, 29-35.

Francis C. A., 1986. Multiple Cropping Systems. MacMillan, N.Y., 383 p.

Guy P., 1965. La compétition intraspécifique chez les plantes fourragères. Fourrages, 22, 22-27.

Haynes R. J., 1980. Competitive aspects of the grass-legume association. Adv. Agron., 33, 227-261.

Jacquard P., 1968. Manifestation et nature des relations sociales chez les végétaux supérieurs. Oecol. Plant., 11, 137-168.

Jacquard P., 1975. Concurrence intraspécifique et potentialités de rendement. Ann. Amélior. Plant., 25 (1), 3-24.

Jacquard P.. 1977. Relations entre espèces dans les associations graminées-légumineuses. Sél. fr., 24, 3-28.

Jonard P., Koller J., Vincent A., 1952, Evolution de la tige et de l'épi chez la variété de bié Vilmorin 27 au cours de la période de reproduction. Ann. Amélior. Plant., 2, 1-24.

Maitre J. P., 1981. Dynamique des populations d'organes et de la production de matière sèche chez Trifolium pratense L. ; rôle de la concurrence. Thèse Doc. Ing., U.S.T.L., Montpellier, 122 p.

Maitre J., Assemat L., Jacquard P., 1985. Croissance du trèfle violet (Trifolium pratense L.) en association avec du ray-grass d'Italie (Lolium multiflorum Lam. ssp. Italicum) : 1) Description de l'organisation morphologique du trèfle, 2) Démographie des organes, bio- masse et interferences biologiques. Agronomie, 5 (3), 251-260 el (4), 297-304.

Nosberger J., 1983. Quelques aspects de la biologie et de la physiologie du trèfle blanc. Fourrages, 94, 49-60.

Ouknider M., 1985. Contribution à l'étude des relations de voisinage dans un peuplement de vesce (Vicia sativa L.)-avoine (Avena sativa L.). D.E.A., U.S.T.L., Montpellier, 53 p.

Ouknider M., Jacquard P., 1986. Production et valeur nutritive de l'association vesce-avoine en zone méditerranéenne. Fourrages, 105, 39-62.

Ross P. J., Henzel F., Ross D. R., 1972. Effects of nitrogen and light in grass-legume pastures : a system analysis approach. $J$. Appl. Ecol., 9, 2, 353-556.

Rotili P., 1979. Contribution à la mise au point d'une méthode de sélection de la luzerne prenant en compte les effets d'interférences entre individus. I. Etude expérimentale de la structure de la luzernière. Ann. Amélior. Plant., 29 (4), 353-381.

Rotili Y., Zannone L., Scotti C., Paoletti R., Gnocchi G., Romani M., 1983. Interferenza interspecifica in piante foraggere. II. Comportamento delle associazioni di erba medica con graminacee in strutture prative costruite a linee alterne e binate. Ann. Ist. spr. Colt. forragere, 6, 71-114.

Spitters C. J. T., 1983. An alternative approach to the analysis of mixed cropping experiments : estimations of competition effects. Neth. J. agric. Sci., 31, 1-11.

Talamucei P., 1976. Influence de la variété de graminćc el de la fumure azolće sur le rendement et l'équilibre de l'association luzerne-dactyle en Toscane. Fourrages, 65, 17-28.

Trenbath B. R., 1974. Biomass productivity in mixtures. $A d v$. Agron., 26, 177-210.

Trenbath B. R., 1976. Plant interactions in mixed crop communities. In : Multiple cropping. R. I. Papendick, P. A. Sanchez, B. B. Triplett (eds), American Society of Agronomy, Madison, Wisconsin, 129-170.

Trenbath B. R., 1978. Models and the interpretation of mixture experiments. In : Plant relations in pastures. J. R. Wilson (ed), CSIRO, Melbourne, 154-162.

Trenbath B. R., 1986. Resource use by intercrops. In : Multiple Cropping Systems. C. A. Francis (ed), MacMillan, N.Y., 57-81.

Wit (de) C. T., 1960. On competition, Versl. Landbouwk. Onderz., $66(8), 1-82$.

Zannone L., Rotili P., Scotti C., Gnocchi G., 1983. Interferenza interspecifica in piante foraggere. I. Associazioni di erba medica con graminacee. Ann. Ist. sper. Colt. foraggere, 6, 25-50.

Zannone L., Paoletti R., Scotti C., Jacquard P., 1985. Grasslegume associations and breeding for yield. Proc. XV Int Grassld. Cong., Kyoto, 207-208.

Zannone L., Rotili P., Paoletti R., Scotti C., 1986. Experimental studies of grass-legume associations. Agronomie, 6 (10), 931-940. 